\section{Case Reports in Nephrology and Dialysis}

Case Rep Nephrol Dial 2021;11:95-102

DOl: $10.1159 / 000512227$

Published online: March 31, 2021

(C) 2021 The Author(s)

Published by S. Karger AG, Basel www.karger.com/cnd

This article is licensed under the Creative Commons Attribution-NonCommercial 4.0 International License (CC BY-NC) (http://www.karger.com/Services/OpenAccessLicense). Usage and distribution for commercial purposes requires written permission.

\title{
Complement-Mediated Thrombotic Microangiopathy Associated with Lupus Nephritis Treated with Eculizumab: A Case Report
}

\author{
Everardo Arias Torres ${ }^{a} \quad$ Yongen Chang $^{\mathrm{b}}$ Sheetal Desai ${ }^{\mathrm{c}} \quad$ Ian Chang \\ Jonathan E. Zuckerman ${ }^{d} \quad$ Richard Burwick $^{\mathrm{e}}$ Kamyar Kalantar-Zadeh $^{\mathrm{b}}$ \\ Ramy M. Hannab \\ ${ }^{a}$ Department of Medicine, University of California, Irvine, CA, USA; ${ }^{b}$ Division of \\ Nephrology, Hypertension and Kidney Transplantation, Department of Medicine, \\ University of California, Irvine, CA, USA; 'Division of Rheumatology, Department of \\ Medicine, University of California, Irvine, CA, USA; dDepartment of Pathology and \\ Laboratory Medicine, University of California, Los Angeles, CA, USA; ${ }^{\text {Department of }}$ \\ Obstetrics and Gynecology, Maternal-Fetal Medicine, Cedars Sinai Medical Center, \\ Los Angeles, CA, USA
}

\section{Keywords}

Thrombotic microangiopathy · Systemic lupus erythematosus · Complement-mediated thrombotic microangiopathy · Glomerular disease · Pregnancy · Atypical hemolytic uremic syndrome

\section{Abstract}

Thrombotic microangiopathies (TMAs) involve multiple organ systems due to the presence of microangiopathic hemolysis. One such condition, atypical hemolytic uremic syndrome (aHUS), is a complement-mediated process that is part of a spectrum of disorders that have underlying complement dysfunction of the alternative pathway due to overactivity or decreased self-nonself discrimination by innate immunity. Complement-amplifying conditions such as pregnancy may unmask a diagnosis of aHUS. We present an important case of a pregnant 23-year-old Hispanic female who presented in mid-gestation (21 weeks) with an initial diagnosis of 


\section{Case Reports in Nephrology and Dialysis}

Torres et al.: aHUS and SLE in Pregnancy

systemic lupus erythematosus (SLE) complicated by aHUS. She met clinical criteria for aHUS on presentation and was found to have a pathogenic CFHR1-3 homozygous deletion. She has been treated with intravenous and oral steroids, cyclophosphamide, subsequently also with plasma exchange, and finally with eculizumab with partial improvement in renal function. This case adds to the emerging literature showing that SLE and aHUS (or complement-mediated TMA) can be successfully treated with $\mathrm{C} 5$ blockade.

(C) 2021 The Author(s)

Published by S. Karger AG, Basel

\section{Introduction}

Thrombotic microangiopathies (TMAs) are a diverse group of diseases presenting with features of microangiopathic hemolytic anemia, thrombocytopenia, and end-organ dysfunction $[1,2]$. Complement-mediated TMA, referred to as atypical hemolytic uremic syndrome (aHUS), is an extremely rare (or possibly rarely recognized) disease caused by deficiencies of complement-regulatory proteins that control the alternative complement system [3, 4]. Patients with alternative complement pathway dysregulation in the form of complement factor $\mathrm{H}(\mathrm{CFH})$ mutations have the worst long-term prognosis, with $73 \%$ progressing to end-stage renal disease within 5 years after diagnosis (in those without anti-factor $\mathrm{H}$ autoantibodies). Since the clinical and laboratory presentation of lupus nephritis with TMA is very similar to aHUS [5, 6], the use of complement inhibition is appropriate in certain settings [7-9].

We present the case of a 23-year-old female with recently diagnosed systemic lupus erythematosus (SLE) in pregnancy who presented with acute renal failure and was found to have diffuse lupus nephritis and TMA on renal biopsy and a large CFHR1-3 homozygous deletion on genetic testing. She underwent eculizumab treatment, resulting in an improvement in kidney function.

\section{Case Presentation}

A 23-year-old Hispanic female, gravida 4 para 1 at 21 weeks' gestation, with recently diagnosed SLE at an outside hospital, was transferred for a higher level of care for rapidly oliguric acute kidney injury (AKI). By Kidney Disease: Improving Global Outcomes (KDIGO) staging, she had stage III AKI with an increase in serum creatinine from baseline of $0.5 \mathrm{mg} / \mathrm{dL}$ to 3.5 $\mathrm{mg} / \mathrm{dL}$ (0.5-1.2 reference range). The patient had no prior diagnosis of SLE before her pregnancy, and she was not on any treatment including steroids, hydroxychloroquine, mycophenolate mofetil, or tacrolimus at the time of initial presentation.

She presented with leukocytosis (white blood cell count of $18,600 / \mu \mathrm{L}$ ), anemia (hemoglobin of $6.4 \mathrm{~g} / \mathrm{dL}$ ), and thrombocytopenia (platelet count of $94,000 / \mu \mathrm{L}$ ), alongside oliguric AKI with hyperkalemia of $5.2 \mathrm{mmol} / \mathrm{L}$ and elevation of blood urea nitrogen at $57 \mathrm{mg} / \mathrm{dL}$ (725). Reticulocytes were $3 \%$, suggesting bone marrow replacement of erythrocytes. The prothrombin time and international normalized ratio was $13.3 \mathrm{~s}$ and 1.01 , respectively. The activated partial thromboplastin time was $42 \mathrm{~s}$. Lactate dehydrogenase was $523 \mathrm{U} / \mathrm{L}$, supporting a suspicion of active hemolysis. Preeclampsia and HELLP (hemolysis, elevated liver enzymes, low platelet count) syndrome were not suspected based on her early gestational age and clinical course without hypertension or elevation of liver enzymes. The liver function test results

\section{Karger'=}




\section{Case Reports in Nephrology and Dialysis}

Case Rep Nephrol Dial 2021;11:95-102

DOI: 10.1159/000512227

c) 2021 The Author(s). Published by S. Karger AG, Basel www.karger.com/cnd

Torres et al: aHUS and SLE in Pregnancy

were as follows: aspartate transaminase, $17 \mathrm{U} / \mathrm{L}$; alanine transaminase, $8 \mathrm{U} / \mathrm{L}$; alkaline phosphatase, $72 \mathrm{IU} / \mathrm{L}$; and total bilirubin, $0.6 \mathrm{mg} / \mathrm{dL}$ (which were within normal limits).

Evaluation of anemia revealed evidence of microangiopathic hemolytic anemia with haptoglobin $<30 \mathrm{mg} / \mathrm{dL}$ (below detection range of assay), presence of 1 or more schistocytes, with normal vitamin $\mathrm{B}_{12}$ and $62 \%$ ADAMTS13 activity ( $\left.>5 \%\right)$. A direct Coombs test was negative. Urinalysis was notable for proteinuria ( $>500 \mathrm{mg} / \mathrm{dL}$ ), but negative for leukocyte esterase and bacteria. There were between 20 and 66 red blood cells/high-power field, but no red blood cell casts captured on manual urinalysis with microscopy done by the treating nephrologists. The SLE was characterized by fatigue, alopecia, oral ulcer, malar rash, pleuropericarditis, positive ANA of 1:320 speckled pattern, high titer positive anti-dsDNA, positive Smith antibody, positive RNP antibody, positive SSA/SSB antibodies, and hypocomplementemia (C3 was low at $45 \mathrm{mg} / \mathrm{dL}, \mathrm{C} 4$ was low at $4 \mathrm{mg} / \mathrm{dL}$, and the total complement level [CH50] was normal at $112 \mathrm{mg} / \mathrm{dL}$ ). Antiphospholipid lipid antibodies (cardiolipin, $\beta_{2}$-glycoprotine, and lupus anticoagulant) were negative.

Hemodialysis was initiated on hospital day 1 for pulmonary edema resulting from oliguric AKI. After extensive discussion with the patient, the decision was made to terminate pregnancy to expedite optimal treatment for SLE. She was managed with methylprednisolone, hydroxychloroquine, and mycophenolate mofetil, with minimal signs of renal recovery and an eventual switch to cyclophosphamide. She subsequently underwent renal biopsy. Figure 1 shows the trend of renal function and platelets versus time.

The renal biopsy (Fig. 2) contained approximately 39 glomeruli (1 globally sclerotic) and demonstrated a diffuse active proliferative glomerulonephritis represented by diffuse endocapillary hypercellularity composed of swollen endothelial cells, leukocytes, frequent neutrophils/karyorrhectic debris, focal necrotizing lesions, focal large cellular crescents ( $\sim 18 \%$ glomeruli involved), and rare large subendothelial (wire loop/hyaline pseudothrombus type) deposits. The majority of capillary loops showed capillary loop double contour formation. There was patchy mild interstitial inflammation and edema as well as acute tubular injury. There was $<10 \%$ interstitial fibrosis and tubular atrophy. There was frequent necrotizing arteriolitis, often at glomerular hila, as well as focal necrotizing arteritis. Arteries also exhibited endotheliosis and focal fibrinoid necrosis. At least one artery exhibited necrotizing vasculopathy without inflammation. Occasional arterioles showed luminal fibrinogen suggestive of thrombi.

Immunofluorescence microscopy demonstrated global granular glomerular capillary wall and mesangial region staining with IgG $(2+)$, IgA $(2+)$, IgM $(2+)$, C1q $(3+), C 3(4+)$, and kappa (1-2+) and lambda (2+) light chains. Tubular basement membranes exhibited patchy staining with IgG and C1q. Arteries exhibited granular mural staining with IgG and C1q. Tissue anti-nuclear antibody staining was present. Arterioles exhibited luminal staining with fibrinogen. At least 2 glomeruli exhibited segmental fibrinogen staining in areas of necrosis. Electron microscopy studies demonstrated finely granular electron-dense (immune complex) deposits in segmental subepithelial, mesangial, intramembranous, and subendothelial locations. There was frequent endothelial separation from basement membranes with interposition of cell processes, electron-lucent flocculent material, rare fibrin tactoids, and very early neomembrane formation associated with segmental mesangiolysis.

The pathologic diagnoses where (a) diffuse lupus nephritis (ISN/RPS class IV) with an activity index of 12 and chronicity index of 1, (b) necrotizing lupus vasculitis, and (c) features suggestive of chronic active TMA. Due to features of TMA on biopsy, an aHUS genetic panel (Machaon Diagnostics) revealed a pathogenic homozygous CFHR1-3 deletion without CFH

\section{Karger'=}




\section{Case Reports in Nephrology and Dialysis}

Case Rep Nephrol Dial 2021;11:95-102

DOI: $10.1159 / 000512227$

Torres et al.: aHUS and SLE in Pregnancy (c) 2021 The Author(s). Published by S. Karger AG, Basel www.karger.com/cnd

autoantibodies. The decision was made for complement inhibition with eculizumab (C5 monoclonal antibody) to target renal-limited TMA, given the poor renal recovery with cyclophosphamide. Figure 1 shows the platelet count and serum creatinine for our presented case.

Her serum creatinine, which peaked at $6 \mathrm{mg} / \mathrm{dL}$, decreased to $3.6 \mathrm{mg} / \mathrm{dL}$ on hemodialysis. This allowed transition to incremental twice-weekly dialysis, showing a partial renal response to the combination of alkylating therapy, continuation of hydroxychloroquine, oral and intravenous corticosteroids, and complement blockade. Prior to complement blockade, the patient had several rehospitalizations for extrarenal complications of lupus, including lupus cerebritis and diffuse alveolar hemorrhage. She was then treated for 1 week with therapeutic plasma exchange 1 month after her initial hospitalization when she presented with diffuse alveolar hemorrhage; at that time, no intravenous immunoglobulin was given. After completion of 7 treatments every 2 weeks of eculizumab, the patient maintained a TMA event-free status, including no decrease in platelet count of $>50 \%$, no plasma exchange, and no recurrence of lupus cerebritis.

\section{Discussion}

The activation of the alternative pathway of the complement system in hemolytic uremic syndrome has been known since 1975 [1-3]. The complement system plays an important part in the adaptive and innate immune systems and consists of more than 40 plasma and membrane-associated proteins [1]. aHUS has been demonstrated to be caused by hyperactivation of the alternative complement pathway with mutations in complement regulators (CFH, CFI, and CFHR proteins), shown to create a predisposition to aHUS. In nearly two-thirds of aHUS cases, there has been a clear mutation in proteins that downregulate the alternative pathway activity (factor $\mathrm{H}$, factor I, membrane cofactor protein, or thrombomodulin), or with an activating mutation in $\mathrm{C} 3$ or factor $\mathrm{B}$ that renders them constitutively overactive [9]. Our patient was homozygous for the pathogenic CFHR1-3 deletion associated with aHUS, which leads to the loss of two genes, CFHR1 and CFHR3, which encode factor H-related proteins 1 and 3, respectively [10]. Pregnancy and SLE are complement-amplifying conditions which may unmask a diagnosis of aHUS, particularly in those with a complement mutation, as seen with our patient. Complement genetic variants, including the homozygous CFHR1-3 deletion, have been reported in $56-64 \%$ of pregnancy-associated aHUS cases, and the diagnosis is often confirmed when the condition worsens rather than improves after delivery $[11,12]$.

As seen in the literature, carriers of CFHR1-3 may remain well for years before presenting with aHUS following an initiating trigger, but our patient had a homozygous CFHR1-3 deletion, which makes it a rare entity with few reported cases. Complete loss of complement regulators CFHR1 and CFHR3 may lead to increased activation of terminal complement pathways [13]. Similar CFH gene mutations have been shown in the literature to accelerate the predisposition to aHUS and the development of lupus nephritis in accurate mouse models (MRL-lpr mice) [8]. Diagnosis of aHUS is done after exclusion of other microangiopathic hemolytic anemias and with possible findings of a pathologic variant mutation or deletion [3]. Traditional therapies include plasma exchange, glucocorticoids, and immune therapies, but recent clinical trials have shown complement inhibition with eculizumab to induce remission of acute episodes of aHUS and prevention of TMA events refractory to plasma therapy.

Eculizumab is a humanized monoclonal antibody that binds to complement protein C5 and inhibits cleavage to $\mathrm{C} 5 \mathrm{a}$ and $\mathrm{C} 5 \mathrm{~b}$, thereby preventing activation of the terminal membrane

\section{Karger'=}




\section{Case Reports in Nephrology and Dialysis}

Torres et al.: aHUS and SLE in Pregnancy

attack complex [9]. The duration of therapy remains controversial, but given the genetic finding of CFHR 1,3 homozygous deletion, the patient will need to remain on complement blockade for a long duration in hope of sustained renal improvement and to prevent extrarenal thromboembolic events [4, 5]. A notable clinical controlled trial by Legendre et al. [14] has shown that eculizumab use in aHUS for both progressive TMA and longstanding TMA results in improvement in renal function, TMA event-free status for at least 12 weeks duration, and improvement in health-related quality of life. Similar trials have shown that early intervention with eculizumab leads to improved clinical outcomes and reversal of organ damage [14].

\section{Conclusions}

Our case emphasizes the need for a high degree of clinical understanding and awareness surrounding complement-mediated TMA [6], as well as an association with pregnancy and SLE as primary drivers of the disease $[7,11]$. Although plasma-based therapeutics can be initiated, clinicians should have a low threshold for moving to terminal-complement inhibition with eculizumab, particularly if ADAMTS-13 activity is detectable.

\section{Statement of Ethics}

Written informed consent from the subject (patient) was obtained. Written consent for publication of the pathology images was also obtained. The work herein conforms with the Declarations of Istanbul and Helsinki. IRB permission was not applied for, as it is not required for individual case reports or case series with 3 patients or less at our institution (University of California Los Angeles).

\section{Conflict of Interest Statement}

R.M.H. and R.B. are paid speakers and consultants for Alexion Pharmaceuticals.

\section{Funding Sources}

K.K.-Z. is supported by the National Institute on Aging of the National Institutes of Health grant R21-AG047036 and the National Institute of Diabetes, Digestive and Kidney Disease grants R01-DK078106, R01-DK096920, U01-DK102163, and K24-DK091419, as well as philanthropist grants from Mr. Harold Simmons and Mr. Louis Chang.

\section{Author Contributions}

E.A.T.: lead writing of manuscript; Y.C.: edited manuscript; S.D. and I.C.: edited manuscript from rheum perspective; J.E.Z.: made pathology figures; R.B.: edited text from OB specialty perspective; K.K.-Z.: edited text; R.M.H.: edited text as senior author from nephrology and TMA perspective.

\section{Karger'=}




\section{Case Reports in Nephrology and Dialysis}

\section{References}

1 Davin JC, van de Kar NC. Advances and challenges in the management of complement-mediated thrombotic microangiopathies. Ther Adv Hematol. 2015 Aug;6(4):171-85.

2 Besbas N, Karpman D, Landau D, Loirat C, Proesmans W, Remuzzi G, et al. A classification of hemolytic uremic syndrome and thrombotic thrombocytopenic purpura and related disorders. Kidney Int. 2006;70(3):423-31.

3 Hanna RM, Hasnain H, Abdelnour L, Yanny B, Burwick RM. Atypical hemolytic uremic syndrome in a patient with protein-losing enteropathy. J Int Med Res. 2019 Aug;47(8):4027-32.

4 Hanna RM, Merin N, Burwick RM, Abdelnour L, Selamet U, Yanny B, et al. Successful use of eculizumab to treat atypical hemolytic uremic syndrome in patients with inflammatory bowel disease. Thromb J. 2019 Sep;17(1):18.

5 Hanna RM, Hanna M, Larson B, Lopez EA, Wilson J, Hendifar A. Thrombotic Microangiopathy due to Catastrophic Antiphospholipid Antibody Syndrome Confirmed on Skin Biopsy and Treated with Eculizumab. J Onconephrol. 2017;1(2):e1-7.

6 Hanna RM, Barsoum M, Vandross A, Kurtz I, Burwick R. Atypical hemolytic uremic syndrome and complement blockade: established and emerging uses of complement inhibition. Curr Opin Nephrol Hypertens. 2019 May;28(3):278-87.

7 Park MH, Caselman N, Ulmer S, Weitz IC. Complement-mediated thrombotic microangiopathy associated with lupus nephritis. Blood Adv. 2018 Aug;2(16):2090-4.

8 Bao L, Haas M, Quigg RJ. Complement factor H deficiency accelerates development of lupus nephritis. J Am Soc Nephrol. 2011 Feb;22(2):285-95.

9 Palma LM, Langman CB. Critical appraisal of eculizumab for atypical hemolytic uremic syndrome. J Blood Med. 2016 Apr;7:39-72.

10 Zipfel PF, Edey M, Heinen S, Józsi M, Richter H, Misselwitz J, et al. Deletion of complement factor H-related genes CFHR1 and CFHR3 is associated with atypical hemolytic uremic syndrome. PLoS Genet. 2007 Mar;3(3):e41.

11 Gupta M, Govindappagari S, Burwick RM. Pregnancy-Associated Atypical Hemolytic Uremic Syndrome: A Systematic Review. Obstet Gynecol. 2020 Jan;135(1):46-58.

12 Gupta M, Feinberg BB, Burwick RM. Thrombotic microangiopathies of pregnancy: differential diagnosis. Pregnancy Hypertens. 2018 Apr; 12:29-34.

13 Heinen S, Hartmann A, Lauer N, Wiehl U, Dahse HM, Schirmer S, et al. Factor H-related protein 1 (CFHR-1) inhibits complement C5 convertase activity and terminal complex formation [C.M.]. Blood. 2009 Sep;114(12):2439-47.

14 Legendre CM, Licht C, Muus P, Greenbaum LA, Babu S, Bedrosian C, et al. Terminal complement inhibitor eculizumab in atypical hemolytic-uremic syndrome. N Engl J Med. 2013 Jun;368(23):2169-81. 


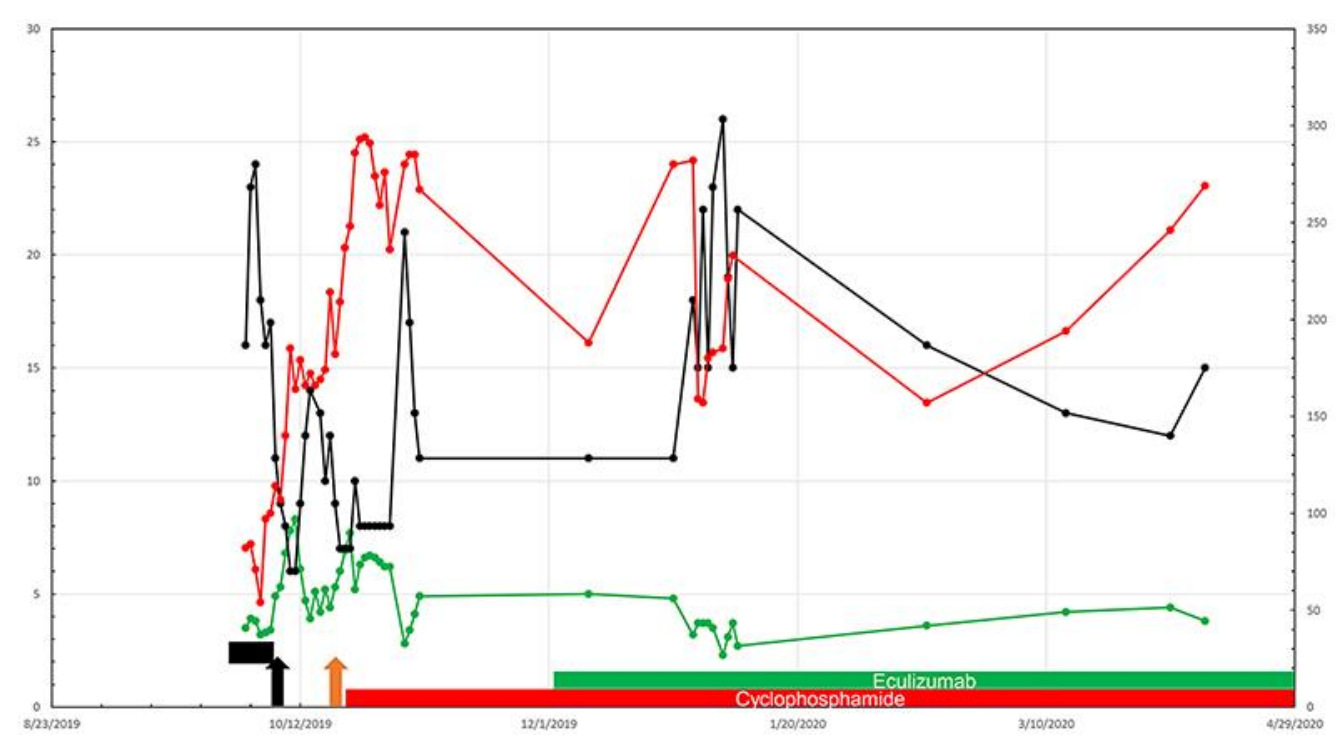

Fig. 1. Trend of serum creatinine $(\mathrm{mg} / \mathrm{dL})$, estimated glomerular filtration rate $(\mathrm{mL} / \mathrm{min})$, and platelet count versus time in the presented patient. Green line = serum creatinine (mg/dL) (left $y$ axis); black line = eGFR $(\mathrm{mL} / \mathrm{min})$ (left $y$ axis); red line = platelet count $(\times 1,000 / \mu \mathrm{L})$ (right $y$ axis); black box = peripartum thrombotic microangiopathy/atypical hemolytic uremic syndrome presentation; black arrow = date of therapeutic abortion; red arrow and box = start and duration of cyclophosphamide treatment; green box = start and duration of eculizumab treatment. 


\section{Case Reports in Nephrology and Dialysis}

\begin{tabular}{l|l}
\hline Case Rep Nephrol Dial 2021;11:95-102 \\
\hline DOI: 10.1159/000512227 & $\begin{array}{l}\text { @ 2021 The Author(s). Published by S. Karger AG, Basel } \\
\text { www.karger.com/cnd }\end{array}$ \\
\hline
\end{tabular}
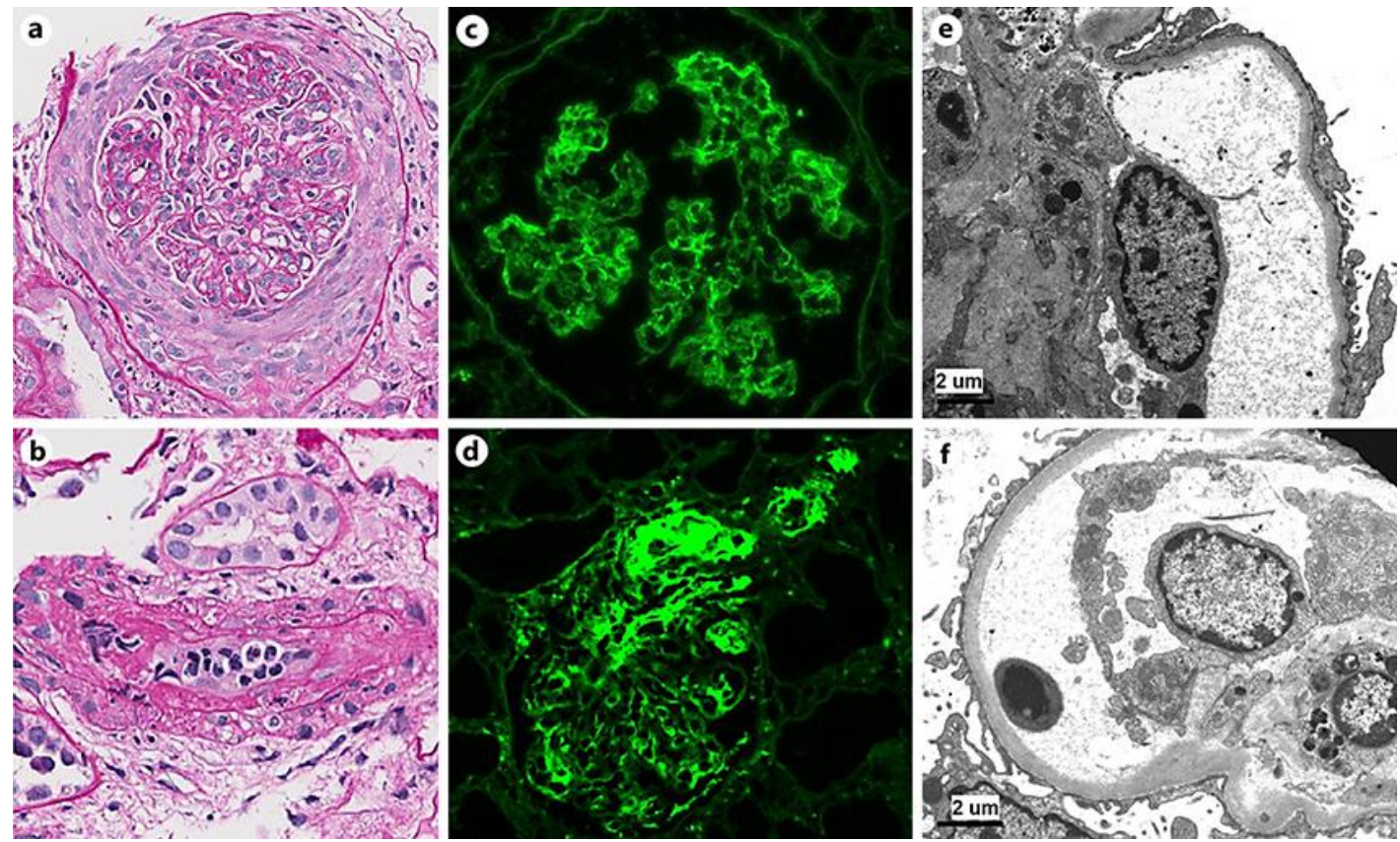

Fig. 2. Renal biopsy findings. a, b Light microscopy images demonstrating global endocapillary hypercellularity and crescent formation (a) and necrotizing arteritis (b). Periodic acid Schiff stain. $\times 400$. c, d Immunofluorescence microscopy images demonstrating a global granular capillary wall and mesangial IgG staining (c) and luminal fibrinogen staining in glomeruli and hilar arterioles (d). $\times 400$. e, $\mathrm{f}$ Electron micrographs demonstrating detachment of endothelial cells from basement membranes with interposition of electronlucent material suggestive of active thrombotic microangiopathy. 